\title{
Reformed permutations in Mousetrap and its generalizations
}

\author{
A.M. BERSANI \\ Dipartimento di Metodi e Modelli Matematici, \\ Università "La Sapienza" di Roma, \\ Via A. Scarpa 16, 00161 Roma, Italy \\ e-mail: bersani@dmmm.uniroma1.it
}

Abstract. We study the reformed permutations in the card game Mousetrap, in Modular Mousetrap and in a solitaire called He Loves Me, He Loves Me $N o t\left((H L M)^{2} N\right)$, which can be considered as a generalization of the classical games Treize and Mousetrap. Thanks to a new technique introduced in a previous paper, we attain new results, giving partial answers to some questions posed by Cayley and by Guy and Nowakowski in their papers.

\section{Introduction}

In 1857 Cayley [3] proposed a card game, called Mousetrap, played with a deck containing only one seed; here we report the description given by Guy [7, p. 237]:

"Suppose that the numbers $1,2, \cdots, n$ are written on cards, one to a card. After shuffling (permuting) the cards, start counting the deck from the top card down. If the number on the card does not equal the count, transfer the card to the bottom of the deck and continue counting. If the two are equal then set the card aside and start counting again from "one". The game is won if all the cards have been set aside, but lost if the count reaches $n+1 . "$

Cayley [4] posed the fundamental question: "Find all the different arrangements of the cards, and inquire how many of these there are in which all or any given smaller number of the cards will be thrown out; and (in the several cases) in what orders the cards are thrown out."

Relatively few authors (Cayley [4], Steen [17], Guy [7], Guy and Nowakowski [8], [9], [10], Mundfrom [11]) studied the problem, arriving, only recently [8], [11], at partial results.

Guy and Nowakowski [7, p. 238], [8], [9] considered another version of the game, called Modular Mousetrap, where, instead of stopping the game when no matching happens counting up to $n$, we start our counting again from "one", arriving to set aside every card or at a loop where no cards can be set aside anymore. Obviously, in this game, if $n$ is prime, we have only two possibilities: derangement or winning deck. Guy and Nowakowski study only few cases $(n \leq 5)$, while in the case $n=6$ they consider only decks starting with an "ace".

The games were studied in the case of only one seed. The author [2] generalized both versions of Mousetrap to the case of several decks ( "multiseed" Mousetrap: $n=m \cdot s$, where $s$ is the number of seeds and $m$ is the maximal value of the cards).

AMS 2000 Subject Classification: 05A05, 05A10, 05A15, 60C05, 65C50

Key words: Combinatorial Analysis, derangement, Mousetrap, reformed permutations 
Let us remark that the "multiseed" Mousetrap was introduced for the first time by the author [2]. We can generalize Mousetrap rules at least in two different ways: when the player has counted up to $m$, without coming to a card which ought to be thrown out, he can

a) either stop the game (Mousetrap-like rule)

b) or eliminate the last $m$ cards and continue his counting, restarting from "one", up to the exhaustion of the deck, when he has eliminated or stored all the cards.

We have chosen the second option, that recalls a different solitaire, commonly called He Loves $\mathrm{Me}, \mathrm{He}$ Loves Me Not $\left((H L M)^{2} N\right)$ or Montecarlo:

"From a deck with $s$ seeds and $m$ ranks, deal one at a time all the cards into a pile, counting "one", "two", "three" etc. When a card whose value is $k$ proves to be of the rank you call, it is hit. The card is thrown out and stored in another pile, the record is increased by $k$, the preceding $k-1$ cards are put at the end of the deck, in the same order in which they were dealt and you start again to count "one", "two", "three" etc. If you have counted up to $m$ without coming to a card has been thrown out, the last $m$ cards are "burned", i.e., definitively discarded and you begin the count afresh, counting "one", "two", "three" etc. with the residual cards. When the number $n_{c}$ of cards in the residual deck is less than $m$, the count can arrive, at most, at the value $n_{c}$. The game ends when you have stored and/or "burned" all the cards and there are no more cards in the deck. The record of the game is given by the sum of the face values of all the stored cards."

Aim of the game is to yield as the greatest record as possible.

Andrea Pompili [12] and the author [1], [2], separately, studied this game, mainly by a numerical point of view, by means of Monte Carlo simulations. The author [2] showed some theoretical results, concerning the probability distribution $P(k)$, where $k$ is the record value. We will recall them in Section 2.

The author [2] introduced a new technique, which allows us to obtain the exact number of winning decks for many values of $m$ and $s$, without any need of simulations, not only for $(H L M)^{2} N$, but also for Mousetrap and Modular Mousetrap, in their "multiseed" version, too. We implemented the technique in a computer program. The new results were obtained in a very efficient way and many others could be reached, if we should implement the algorithm in a parallel calculus framework.

In their papers, Guy and Nowakowski proposed to study the so-called reformed decks (or permutations): "consider a permutation for which every number is set aside. The list of numbers in the order that they were set aside is another permutation. Any permutation obtained in this way we call a reformed permutation. Characterize the reformed permutations."

Aim of this paper is to apply the new technique to the analysis of reformed decks, in the three games and to show new results, which can answers to some open questions proposed by Cayley and by Guy and Nowakowski.

The paper is divided into five Sections.

In Section 2 we recall the most important theoretical results for the three games.

In Section 3 we briefly describe the technique introduced by the author [2] and show the first results attained at $(H L M)^{2} N$.

In Section 4, applying the technique to the study of reformed decks in the three games, we obtain many (even unexpected and curious) results.

In Section 5 we present a short review of open problems and perspectives. 


\section{Preliminary results on Mousetrap and $(H L M)^{2} N$}

For all the games considered in this paper there are few results.

Cayley [3] proposed to investigate, at Mousetrap, whatever the number $n$ of cards is, which permutations throw out the cards in the same order of their numbers. He obtained the corresponding permutations for $n \leq 8$ :

$$
1 ; 12 ; 132 ; 1423 ; 13254 ; 142563 ; 1527436 ; 16245378 \text {. }
$$

Guy and Nowakowski observed that not all the permutations are reformed permutations. On the other hand, the identity permutation $12 \cdots n$ is always a reformed permutation. Since it is not possible, in general, to arrange the cards so that all the cards may be thrown out in a predetermined order, Cayley [4] posed the following questions:

1) for each $n$ find the winning permutations of $12 \cdots n$;

2) for each $n$ find the number of permutations that eliminate precisely $i$ cards for each $i, 1 \leq i \leq n$.

He studied the game Mousetrap in the case $n=4$, analyzing the $4 !=24$ different decks. Curiously, he made mistakes in six decks.

Steen [17], already in 1878 and, much more recently, Guy and Nowakowski [8] and Mundfrom [11], obtained deeper results. Steen calculated, for any $n$, the number $a_{n, i}$ of permutations that have $i, 1 \leq i \leq n$, as the first card set aside and the numbers $b_{n, i}$ and $c_{n, i}$ of permutations that have "one" (respectively "two") as the first hit and $i$ as the second. He obtained the following recurrence relations:

$$
\begin{gathered}
a_{n, 1}=(n-1) ! \quad, \quad a_{n, i}=a_{n, i-1}-a_{n-1, i-1} \quad, \quad b_{n, i}=a_{n-1, i-1} \quad, \quad \forall i=2, . ., n \\
c_{n, i}=c_{n, 1}-(i-1) c_{n-1,1}+\sum_{k=2}^{i-2}(-1)^{k} \cdot \frac{i(i-1-k)}{2} c_{n-k, 1} \quad \forall n>i+1
\end{gathered}
$$

Denoting with $a_{n, 0}$ the number of derangements; $a_{n}=\sum_{k=1}^{n} a_{n, k}$ the total number of permutations which give hits; $b_{n, 0}$ the number of permutations giving "one" as the only hit; $b_{n}=\sum_{k=2}^{n} b_{n, k}$ the total number of permutations giving a second hit, "one" being the first; $c_{n, 0}$ the number of permutations giving "two" as the only hit; $c_{n}=\sum_{k=1}^{n} c_{n, k}(k \neq 2)$ the total number of permutations giving a second hit, "two" being the first; putting $a_{0,0}=1$, Steen showed that, for $0 \leq i \leq n$

$$
\begin{gathered}
a_{n, 0}=a_{n+1, n+1} \quad, \quad a_{n, 0}=n a_{n-1,0}+(-1)^{n} \quad, \quad a_{n, i+1}=\sum_{k=0}^{i}(-1)^{k}\left(\begin{array}{l}
i \\
k
\end{array}\right)(n-1-k) ! \\
b_{n, i}=a_{n-1, i-1}=a_{n-2, i-2}-a_{n-3, i-2} \quad, \quad b_{n, 0}=a_{n, 1}-b_{n}=a_{n, 1}-a_{n-1}=a_{n-1,0} \\
a_{n}=n a_{n-1}+(-1)^{n-1} \quad, \quad b_{n}=a_{n-1} \\
c_{n, i}=\left[\sum_{k=1}^{i-3}(-1)^{k+i-1} \frac{k(k+3)}{2}(n-i+k-1) !\right]-(i-1)(n-3) !+(n-2) !
\end{gathered}
$$


Guy and Nowakowski [8] and Mundfrom [11] showed separately that Steen's formula (2.6) is not valid for $i=n-1$ and $i=n$ and gave the exact relations. We quote the expressions, together with the equation for $c_{n}=\sum_{k=1}^{n} c_{n, k}, k \neq 2$, as shown by Guy and Nowakowski [8], thanks to their compactness:

$$
\begin{gathered}
c_{n, n-1}=\sum_{k=0}^{n-3}(-1)^{k}\left(\begin{array}{c}
n-3 \\
k
\end{array}\right)(n-k-2) ! \\
c_{n, n}=(n-2) !+\left[\sum_{k=0}^{n-5}(-1)^{k+1}\left(\left(\begin{array}{c}
n-4 \\
k
\end{array}\right)+\left(\begin{array}{c}
n-3 \\
k+1
\end{array}\right)\right)(n-k-3) !\right]+2(-1)^{n-3} \\
c_{n}=(n-2)(n-2) !-\left[\left[\begin{array}{c}
1 \\
e
\end{array}((n-1) !-(n-2) !-2(n-3) !]\right],\right.
\end{gathered}
$$

where $[[x]]$ is the nearest integer to $x$.

Steen [17], Guy and Nowakowski [8] and Mundfrom [11] elaborated some tables related to formulas (2.1) - (2.9). The sequences there reproduced are quoted by Sloane [13], [14], [15] in the following way:

$\left\{a_{n}\right\}_{n \in \mathbb{N}}([\mathbf{1 7}]):$

$\left\{a_{n, 0}\right\}_{n \in \mathbb{N}}([\mathbf{1 7}]):$

$\left\{a_{n, 2}\right\}_{n \geq 2}([\mathbf{1 7}]):$

$\left\{c_{n}\right\}_{n \geq 2}([\mathbf{8}],[\mathbf{1 1}],[\mathbf{1 7}]):$

$\left\{c_{n, 0}\right\}_{n \geq 2}([\mathbf{1 1}],[\mathbf{1 7}]):$

$\left\{c_{n, 3}\right\}_{n \geq 3}([\mathbf{1 1}],[\mathbf{1 7}]):$

$\left\{c_{n, 4}\right\}_{n \geq 4}([\mathbf{1 1}],[\mathbf{1 7}]):$

$\left\{c_{n, 5}\right\}_{n \geq 5}([\mathbf{1 1}],[\mathbf{1 7}]):$

$\left\{c_{2,1}\right\} \cup\left\{c_{n, n}\right\}_{n \geq 3}([\mathbf{1 1}],[\mathbf{1 7}])$ :
[13] N1423, [14] M3507, [15] A002467;

[13] N0766, [14] M1937, [15] A000166;

[13] N1436, [14] M3545, [15] A001563;

[13] N1186, [14] M2945, [15] A002468;

[13] N1635, [14] M3962, [15] A002469;

[15] A018931;

[15] A018932;

[15] A018933;

[15] A018934.

Let us observe that, owing to his mistakes in the formula for $c_{n, i}$, Steen reported erred sequences for $\left\{c_{n}\right\}_{n \geq 2},\left\{c_{n, 0}\right\}_{n \geq 2}$ and $\left\{c_{2,1}\right\} \cup\left\{c_{n, n}\right\}_{n \geq 3}$. The correct sequences, obtained by Mundfrom, are quoted as [15] A002468, A002469 and A018934.

Guy and Nowakowski [8] provided the correct form of the sequence [15] A002468. Sequences [15] A000166 of derangements $\left\{a_{n, 0}\right\}$ and A002467 of permutations with at least one fixed point arrive at $n=21$, but can be easily improved by means of the following Proposition (see, for example, [2]):

Proposition 2.1. The probability of derangement for the games Mousetrap (M) and Modular Mousetrap $(M M)$ is

$$
P_{M, m}(0)=P_{M M, m}(0)=\sum_{k=0}^{m} \frac{(-1)^{k}}{k !} .
$$

and

$$
\lim _{m \rightarrow \infty} P_{M, m}(0)=\lim _{m \rightarrow \infty} P_{M M, m}(0)=P o_{1}(0)=e^{-1},
$$

where $P o_{1}(k)$ is the poissonian distribution with characteristic parameter 1: $P o_{1}(k)=\frac{e^{-1}}{k !}$. 
As a partial answer to question 2) by Cayley, Guy and Nowakowski [8] produced a table, giving the numbers of permutations eliminating just $i$ cards $(1 \leq i \leq 9)$; the diagonal represents the numbers of winning permutations, i.e., permutations setting aside all the $n$ cards and represents a partial answer to question 1) by Cayley. Guy and Nowakowski computed the terms up to $n=9$. Since the table does not derive from any closed formula, it was probably obtained by means of direct computations, considering that, for $n=9$, it is possible to check, by means of a computer, all the permutations, whose number is equal to $9 !=362880$.

This table is quoted as [15] A028305, but only up to $n=7$.

We can derive other sequences from this table: the first column is the sequence [15] A000166 of derangements. The second column is the sequence [15] A007710 ([14] M1695) of permutations eliminating just one card. The top diagonal is the sequence [15] A007709 ([14] M1608) of winning (or reformable) decks, i.e., of decks eliminating all the cards. The sums of the terms of each row, except the terms on the top diagonal, give the first nine terms of the sequence [15] A007711 ([14] M3546) of unreformed decks, i.e., of decks which do not eliminate all the cards.

Furthermore, Guy and Nowakowski proved the formula for the probability that only the card with value $k$ is set aside from a deck of $n>2$ cards and showed the related complete table of values, for $1 \leq k \leq n, 1 \leq n \leq 10$, adding another table, for $11 \leq n \leq 17$, but $1 \leq k \leq 5$.

Sequence [15] A028306 quotes the table, but only up to $n=8$.

Knowing general formulas giving the numbers of permutations that have $i$ as the $k$-th hit, given the previous $(k-1)$ hits, would be very useful to arrive at a closed formula for the probability distribution of the game. But, as remarked by Steen, already the computations to obtain $c_{n, i}$ are very difficult and it is hard to expect more advanced results in this direction.

In Section 4 we will show new results, based not on closed formulas, but on computer aided analysis, which can extend the results attained by Guy and Nowakowski [8] up to $m=15, s=1$ and to "multiseed" Mousetrap.

Finally, Guy and Nowakowski [8] yielded some results for the game Modular Mousetrap. Their paper is mostly focussed on the problem of reformed permutations, i.e., decks obtained as lists of cards in the order they were set aside in a winning deck. We will take into consideration this problem in Section 4.

Concerning $(H L M)^{2} N$, since, after every matching, we start counting again from "one", the game recalls Mousetrap, but differs from it for the following reasons:

a) we record the sum of the values of the cards, not their number; obviously, in a deck of $m \cdot s$ cards, we can, at most, obtain

$$
s \cdot \sum_{k+1}^{m} k=\frac{s}{2} m(m+1) \quad \text { points ; }
$$

b) we "burn", i.e., we eliminate $m$ cards, if no coincidences occur counting from 1 to $m$, but we do not stop the game and we continue our counting starting again from "one".

We can stop the game either when, remaining in the deck a number $n_{c}<m$ of cards, we do not obtain any matching counting up to $n_{c}$, or, following $T 4$ and Mousetrap rules, when the deck falls short, we turn the deck of $n_{c}$ cards and we continue our counting up to $m$; in this second case, if no matching happens counting up to $m$, we stop the game; otherwise we can restart the counting, after having stored the last matching card. In the first case, we play $(H L M)^{2} N$; in the second we play the "multiseed" Mousetrap. 
Let us observe that the rule followed by Mousetrap allows the player to store all the $m \cdot s$ cards (in particular, in Mousetrap, if we remain with only one card in the deck, we know that we will store it, because we will count up to $m$ visiting always the same card, whose values is, obviously, less or equal to $m$ ). Instead, as shown in the following Proposition, in $(H L M)^{2} N$ we can store at most $m s-1$ cards. In other words, when we consider Mousetrap with more than one seed, this game is easier than $(H L M)^{2} N$ and every deck winning at $(H L M)^{2} N$ wins at Mousetrap.

Proposition 2.2.([2]) In $(H L M)^{2} N$, for every $s, m$ we can at most store $m s-1$ cards and the record cannot exceed

$$
C_{\text {max }}:=\frac{s}{2}[m(m+1)]-2 .
$$

The crucial question is if it is always possible to find a deck from which we can store all the decks but a "two" and, consequently, we can obtain $C_{\max }$.

The author [1] stated the following two conjectures:

Strong Conjecture (SC) For every $s \geq 3 ; m \geq 2$, there exists at least one deck from which we store $s m-1=n-1$ cards, obtaining the best record, i.e.,

$$
C_{\text {max }}=\frac{s}{2} m(m+1)-2 .
$$

Weak Conjecture (WC) For every $s \geq 2 ; m \geq 2$, there exists at least one deck from which we store sm $-1=n-1$ cards.

Let us observe that, for $s=1$, the only way to store the card with value $m$ consists in putting it in the $m$-th place, without having any other coincidences in the previous $(m-1)$ places. Let us indicate with $X_{1} X_{2} X_{3} \ldots X_{m-2} X_{m-1}$ an arbitrary derangement of the first $(m-1)$ cards; thus the $m$ cards have the following sequence in the deck:

$$
X_{1} X_{2} X_{3} \quad \ldots \quad X_{m-2} X_{m-1} m .
$$

But in the turn following the matching of the card $m$, the residual deck is formed by $(m-1)$ cards, placed in a derangement; consequently we cannot have any other coincidences. Thus for $s=1$ (SC) is false.

Proposition 2.3.([2]) Formula (2.11) is not valid for $s=1$.

Due to the possibility to turn the deck and, consequently, to the fact that every matching is strongly dependent on the previous events, the usual theory of probability distributions for matching problems, as the classical approximation theory, based on binomial or poissonian distributions, does not hold anymore. In fact, a matching occurring not at the first turn is affected by the event that, in the previous turns, the card has not matched any counting.

However, we can give the exact value of the derangement probability, by means of very general results by Fréchet.

Proposition 2.4.([2], [6]) The probability $P_{(H L M)^{2} N, m s}(0)$ of derangement in the game $(H L M)^{2} N$ is

$$
P_{(H L M)^{2} N, m s}(0)=\sum_{k=0}^{m s}(-1)^{k} \frac{(m s-k) !}{(m s) !} \sum_{\sum a_{j}=k}\left[\prod_{j=1}^{m} a_{j} !\left(\begin{array}{c}
s \\
a_{j}
\end{array}\right)^{2}\right] .
$$

and

$$
\lim _{m \rightarrow \infty} P_{(H L M)^{2} N, m s}(0)=P o_{s}(0)=e^{-s} .
$$




\section{The "eulerian" approach.}

Very recently, the author [2] introduced a new technique, which yields, in a very efficient way, not only a positive answer to (SC) at least up to $m=13, s=4$, i.e., for the classical deck of French cards (though it can be used to explore much larger decks), but also the exact number of winning decks (see Table 14) and consequently the exact probability of winning decks, or winning probability, $P_{\max }:=P\left(C_{\max }\right)$, i.e., the probability to obtain the best record, for a large number of cases, as shown in [2, Table 5].

The technique, implemented in a FORTRAN program, rebuilds strings of more and more increasing length (up to the winning strings of length $n$, or $n$-strings), storing in data files only those ones such that the sub-decks, rebuilt from them, win playing $(H L M)^{2} N$, i.e., store all the cards but the final "two". The program, starting from a $k$-string, read in a data file, builds all the $(k+1)$-strings, obtained adding at the beginning of the actual $k$-string all the allowed values from 1 to $m$; rebuilds the corresponding sub-decks; plays with the sub-decks; if a sub-deck sets aside all the cards, but a "two" and generates the original string, the program stores the corresponding winning $(k+1)$-string.

In order to save disk usage, we store the strings as "characters" in the FORTRAN data files. Every idea about any further memory saving improvement would be welcome.

More precisely, the algorithm is the following: starting from the last "two", we proceed backward, building all the sub-strings, of increasing length, that can guarantee the storing of all the cards, apart from the last "two". Obviously, the last stored card can be only an "ace" or a "two"; similarly, the last but one can be only an "ace" or a "two": the drawing of a "three" as the last but one stored card is excluded by the considerations related to Proposition (2.3). Continuing our reasoning, the last but two stored card can be only an "ace", a "two" or a "three", the last but three an "ace", a "two", a "three" or a "four" and so on, up to the last but $(m-1)$ stored card, which cannot assume a value greater than $(m-1)$. From the last but $m$ stored card on, every card value is admitted.

Practically, let us recall that in the winning $n$-strings the last position must be occupied by a card whose value is "two" and that the strings of length $k \leq m$ (or $k$-strings) cannot be occupied by a card whose value is greater or equal to $k$; consequently, in order to have winning strings, the position just before the last "two" can be occupied only by an "ace" or a "two"; thus we have only two winning final strings of length two: 12 and 22 , which are respectively generated by the sub-decks 12 and 22 .

The final strings of length three can be four: $112 ; 212 ; 122 ; 222$. Clearly, the choice of these strings is related to $s$. If, for example, $s=2$, we must exclude the fourth string, because it contains three identical cards.

Each one of these strings is in a one-to-one correspondence with a sub-deck generating it. In fact

from the string 112 we build the sub-deck 112 , which generates the string 112 ;

from the string 212 we build the sub-deck 221 , which generates the string 212 ;

from the string 122 we build the sub-deck 122 , which generates the string 122 ;

from the string 222 we build the sub-deck 222 , which generates the string 222 .

When we pass to the final strings of length four we have 12 possibilities:

$1112 ; 2112 ; 3112 ; 1212 ; 2212 ; 3212 ; 1122 ; 2122 ; 3122 ; 1222 ; 2222 ; 3222$.

While we can associate to eight of them the corresponding generating winning deck, according to the 
following list:

$$
\begin{aligned}
& \text { the sub - deck } 1112 \text { generates the string } 1112 \text {; } \\
& \text { the sub-deck } 2211 \text { generates the string } 2112 \text {; } \\
& \text { the sub - deck } 1221 \text { generates the string } 1212 \text {; } \\
& \text { the sub- deck } 2132 \text { generates the string } 3212 \text {; } \\
& \text { the sub - deck } 1122 \text { generates the string } 1122 \text {; } \\
& \text { the sub- deck } 2212 \text { generates the string } 2122 \text {; } \\
& \text { the sub - deck } 1222 \text { generates the string } 1222 \text {; } \\
& \text { the sub-deck } 2222 \text { generates the string } 2222 \text {; }
\end{aligned}
$$

we realize that the strings $3112 ; 2212 ; 3122 ; 3222$ have no corresponding winning deck. In fact, considering, for example, the string 3122 , the deck generating it must have in the third position the card "three"; in the fourth position the card "ace" and, having no other components after, the second "ace" must be put in first position. Consequently, we must put the card "two" in the unique place remained, that is in the second position. So the generating deck should be 1231 . But it is evident that this deck, instead of the considered string, generates the loosing string formed only by an "ace", without any other coincidences.

Moreover, to the string 2212 corresponds the deck 1222 , which generates the string 1222 , which is still a winning string, but different from the original one. This last consideration shows that there is no bijective correspondence between decks and strings; if every deck generates only one string, the reverse is in general not true: the same deck can be rebuilt from different strings!

In order to avoid these situations, the algorithm we have implemented contains a test, where we check if the original string coincides with the reformed string, obtained from the deck given back by the original string. Otherwise we must discard the string.

Continuing the procedure, we select winning strings of more and more increasing length, with the fundamental restriction that they must be generated by a deck, following the rules of $(H L M)^{2} N$.

We can interpret the way to deal the cards one after the other as an eulerian description of the game, since the observer is fixed and all the elements of the system, i.e., the cards, pass in front of it, while in the numerical schemes another description, consisting in arranging sequentially all the cards and visiting them by means of a cursor, can be interpreted as a lagrangian description, because in this case we follow the evolution in time of the whole system, starting from its initial configuration.

The operation of rebuilding decks starting from strings of cards put in the same order in which they were set aside can be seen as the reverse operation of the "eulerian" description of the game. So we will speak about "eulerian" technique and "eulerian" strings.

By virtue of this technique we have been able to show that (SC) is true at least up to the case of French cards $(m=13, s=4)$, finding, in less than one second, four winning decks. The first winning deck of French cards found by the computer is the following:

$\begin{array}{ccccccccccccc}7 & 9 & 5 & 9 & 7 & 3 & 8 & 6 & 6 & 2 & 5 & 12 & 11 \\ 4 & 12 & 9 & 7 & 7 & 10 & 2 & 4 & 5 & 3 & 11 & 13 & 2 \\ 4 & 4 & 11 & 13 & 3 & 6 & 10 & 10 & 10 & 3 & 5 & 12 & 2 \\ 1 & 1 & 1 & 1 & 12 & 9 & 11 & 13 & 8 & 8 & 6 & 8 & 13\end{array}$


while the first deck of Italian cards $(m=10, s=4)$ is

$\begin{array}{cccccccccc}6 & 8 & 9 & 7 & 5 & 5 & 3 & 6 & 6 & 10 \\ 2 & 7 & 4 & 7 & 4 & 10 & 2 & 8 & 5 & 3 \\ 9 & 2 & 4 & 4 & 3 & 6 & 10 & 7 & 10 & 3 \\ 5 & 2 & 1 & 1 & 1 & 1 & 9 & 9 & 8 & 8\end{array}$

The author [2] applied this method to prove the following

Proposition 3.1.For $s=2, m=3,4,5$ there are no winning decks. For $s=2, m=6$ there exists only one winning deck.

The search for at least one winning deck is, in general, very fast. On the other hand, the search for all the winning decks is much more complicated, mainly because of disk usage problems.

Table 14 shows all the results attained up to now.

\section{Searching for reformed decks}

As already remarked in the Introduction, there are few results related to the game Mousetrap (M). In particular, there are no (even approximated) formulas giving the probability of winning decks or winning probability $P_{M, m \cdot s}(m \cdot s)$. The technique introduced in the previous Section, adequately adapted to this game, allows us to obtain not a closed formula, but a sequence of values, giving the number $N_{M, m \cdot s}$ of winning decks and, consequently, the winning probability $P_{M, m \cdot s}(m \cdot s)$ for several values of $m$ and $s$. Up to now, according to Chua [5] and Sloane [13], [14], [15], the sequence of values of $N_{M, m \cdot s}$ was obtained only for $s=1$ and up to $m=n=13$. The updated sequence cannot be read in [15] A007709, but can be easily obtained from A007711, i.e., the sequence of non-winning decks (or unreformed decks), because their number is, obviously, equal to $n !-N_{M, m \cdot s}$.

According to Kok Seng Chua [5], this sequence has been obtained playing with all the $n !=m !$ decks, by means of a computer program generating all the permutations of a set of $n$ elements and of parallel calculus, due to the very high number of trials to be performed.

Our new technique allows us to obtain the same results very quickly and to extend the sequence, for $s=1$, up to $m=15$, without any aid of parallel calculus. For example, my PC, equipped with a Pentium IV, yielded the exact number of winning 13-decks in less than 2 hours, in comparison with one week job of parallel calculus used by K. S. Chua [5].

The new sequence of reformed decks (starting from $n=1$ ), quoted by Sloane [15] as A007709, is thus

$$
1 ; 1 ; 2 ; 6 ; 15 ; 84 ; 330 ; 1,812 ; 9,978 ; 65,503 ; 449,719 ; 3,674,670 ; 28,886,593 \text {; }
$$

$266,242,729 ; 2,527,701,273$

while the sequence of unreformed decks (i.e., the total number of non winning decks), quoted as A007711, is now

$$
\begin{aligned}
& \mathbf{0} ; \mathbf{1} ; \mathbf{4} ; \mathbf{1 8} ; \mathbf{1 0 5} ; \mathbf{6 3 6} ; \mathbf{4}, \mathbf{7 1 0} ; \mathbf{3 8 , 5 0 8} ; \mathbf{3 5 2 , 9 0 2} ; \mathbf{3}, \mathbf{5 6 3}, \mathbf{2 9 7} ; \mathbf{3 9}, \mathbf{4 6 7 , 0 8 1} \\
& \mathbf{4 7 5}, \mathbf{3 2 6}, \mathbf{9 3 0} ; \mathbf{6}, \mathbf{1 9 8}, \mathbf{1 3 4}, \mathbf{2 0 7} ; 86,912,048,471 ; 1,305,146,666,727
\end{aligned}
$$

(the values in boldface were already quoted by Sloane [15] or Chua [5]).

Remark 4.1. Though we were not interested on the original question by Cayley [3] ("investigate, whatever the number $n$ of cards is, which permutations throw out the cards in the same order of their 
numbers"), the "eulerian" technique can be easily adapted to check if any particular permutation is a reformed deck. In fact, our technique starts just from a permutation and tries to rebuild the deck from which the permutation is reformed. In particular, we could very easily yield, for every $n$, the deck producing as reformed permutation the identity $12 \cdots n$.

We adapted the "eulerian" technique to the game Modular Mousetrap (MM), too. Though experimentally $N_{M M, m \cdot s}$ grows much faster than $N_{M, m \cdot s}$, the new technique has proved to be very powerful, as shown in the Tables 7 and 8.

Furthermore, we have obtained a huge amount of results in the "multiseed" Mousetrap $(s>1)$, arriving, just as a test of the efficiency of the new technique, to $s=2, m=8 ; s=3, m=6 ; s=4, m=5$ for Mousetrap and to $s=1, m=13 ; s=2, m=7 ; s=3, m=5 ; s=4, m=4$ for Modular Mousetrap.

Let us remark that Guy and Nowakowski [8] studied Modular Mousetrap only in the cases $s=1, m \leq 5$, while in the case $s=1, m=6$ they studied only the decks where the first card is an "ace".

Our results are shown in Tables $1-8$ and can be extended to the cases $s>4$ and, by means of parallel calculus, to higher values of $m$.

Remark 4.2. Guy and Nowakowski [8] observed that, at Modular Mousetrap, when $s=1$ and $m$ is prime, every deck which is not a derangement is a winning deck, because the cards have no possibilities to end in a loop. Consequently, while, if $m$ is not prime, there is no a priori rule showing what is the winning probability, Table 5 shows that, when $s=1$ and $m$ is prime, it is very easy to know the exact winning probability:

$$
P_{M M, m \cdot 1}(m)=1-P_{M M, m \cdot 1}(0) \quad \forall m \text { prime } .
$$

Thus, knowing the sequence [15] A002467 of permutations with at least one fixed point, we immediately obtain the sequence of numbers of winning decks, for $n$ prime:

$3,936,227,868$ for $n=m=13 \quad ; \quad 224,837,335,816,336$ for $n=m=17 \quad ; \quad 76,894,368,849,186,894$ for $n=m=19$ and so on.

For these cases the "eulerian" technique should have proved to be computationally too much expensive, for a single PC.

Furthermore, since $\lim _{n m \rightarrow \infty} P_{M M, m \cdot 1}(0)=\frac{1}{e} \sim 0.367879441$, it follows that, at Modular Mousetrap, $\lim _{m \rightarrow \infty} P_{M M, m \cdot 1}(m)=1-\frac{1}{e} \sim 0.632120559$, if we consider only the sequence of prime numbers $m$.

For the other numbers, the winning probability seems to oscillate and tend to zero very slowly, when $m \rightarrow \infty$.

Finally, the new technique has proved to be extremely efficient when applied to the study of reformed decks (or reformed permutations): as already recalled, when a deck wins at Mousetrap, Modular Mousetrap or $(H L M)^{2} N$, it generates a new deck, which is called reformed deck. We can play again with this new deck, in order to check if it will win again.

When we can repeat this operation $k$ times, we will define $k$-times reformable deck the original deck and $k$-reformed deck the permutation obtained in the $k$-th reformation.

A $k$-cycle is a reformed deck where the $k$-th reformation coincides with one of the previous reformations (not necessarily the original deck). We will classify the cycles more clearly in the second part of this Section, devoted to Modular Mousetrap. 
Guy and Nowakowski [8] firstly proposed the study of reformed decks, posing the following questions: 3) characterize the reformed permutations;

4) for a given $n$, what is the longest sequence of reformed permutations?

5) are there sequences of arbitrary length? are there any cycles other than

$$
1 \rightarrow 1 \rightarrow 1 \ldots \quad \text { and } \quad 12 \rightarrow 12 \rightarrow 12 \ldots ?
$$

6) in Modular Mousetrap are there $k$-cycles for every $k$ ? what is the least value of $n$ which yields a $k$-cycle?

Playing Mousetrap, they obtained only partial results. In particular they investigated only the cases $s=1, m \leq 9$. They yielded at most 3-reformed decks and did not find any non trivial deck.

K. S. Chua [5] achieved a substantial improvement for the game Mousetrap, finding for the first time a 4-reformed deck, in the case $s=1, m=11$. His results are quoted by Sloane [15] in the sequences A007711, A007712, A055459, A067950. As far as I know the sequence of the numbers of 4-reformed decks is not yet quoted in [15].

Here we further improve the above mentioned results, extending the sequences A007711, A007712, A055459, A067950 up to $m=15$ for Mousetrap, with the main goal to find at least a 5-reformed deck. Up to now, there is no evidence of $k$-reformed decks, with $k>4$. With the new results reported in Table 1 , the sequence of numbers of 4 -times (but not 5 -times) reformable permutations is

$$
0,0,0,0,0,0,0,0,0,0,2,1,1,4,14 \text {. }
$$

The discovery of a 5-reformed permutation could represent an important step in the direction of a positive answer to question $\mathbf{5}$ ).

The actual absence of 5-reformed decks must be related to the probability $P_{M, m \cdot s}(m \cdot s)$, given by the ratio between the number of reformed decks and the number of all the permutations of the deck.

We can have a rough estimate of the number of $k$-reformed decks multiplying the number of $(k-1)$-times reformable permutations by $P_{M, m \cdot s}(m \cdot s)$.

Obviously, the probability to obtain a reformed deck is, in general, not equal to the probability to obtain, from all the reformed decks, a 2-reformed one and, in general, from all the decks reformed at least $k$ times, a $(k+1)$-reformed one. But, empirically, all these probabilities are comparable. For example, indicating with $N_{\geq k, m \cdot s}$ the number of decks which are at least $k$-reformable, we have, in the case $s=1, m=15$,

$$
\begin{aligned}
& P_{M, 15 \cdot 1}(15)=\frac{N_{\geq 1,15 \cdot 1}}{15 !} \sim 0.001933 \quad ; \quad \frac{N_{\geq 2,15 \cdot 1}}{N_{\geq 1,15 \cdot 1}} \sim 0.001930 ; \\
& \frac{N_{\geq 3,15 \cdot 1}}{N_{\geq 2,15 \cdot 1}} \sim 0.001991 \quad ; \quad \frac{N_{\geq 4,15 \cdot 1}}{N_{\geq 3,15 \cdot 1}} \sim 0.001442 .
\end{aligned}
$$

Multiplying the numbers $N_{\geq k, 15 \cdot 1}$ by $P_{M, 15 \cdot 1}(15) \sim 0.0019$, the estimates for the numbers of $k$-reformed decks are very close to the real ones, reported in Table 1 . The estimate 0.0266 , obtained multiplying the number of 4-reformed decks by $P_{M, 15 \cdot 1}(15)$, is still too much small to expect a 5-reformed deck.

In the case $m=11, s=1$, when the first 4-reformed decks were yielded, the winning probability is $P_{M, 11 \cdot 1}(11) \sim 0.011$ and multiplying the number of at least 3-reformed decks for this value, we obtain the estimate 0.6 , which is sufficiently high to guarantee the appearance of 4-reformed decks.

Clearly, if we know the exact value of $P_{M, m \cdot s}(m \cdot s)$, we are already able to give the exact number of $k$-reformed decks. 
But when we have no information on the number of $k$-reformed decks, knowing an estimate of $P_{M, m \cdot s}(m$. $s$ ) could allow us to predict for which value of $n$ we can expect the first 5 -reformed decks. Analyzing the evolution of the values of $P_{M, m \cdot 1}(m)$ and of $N_{\geq 4, m \cdot 1}$ for $m \leq 15$, we can make a prediction on the order of number of $k$-reformed decks, for values of $n$ that we cannot yet study with our technique.

Our prediction strongly depends on the competition between the growth rate of $N_{M, m \cdot s}$ and the decrease rate of $P_{M, m \cdot s}(m \cdot s)$.

The winning probability $P_{M, m \cdot 1}(m)$ decreases, for increasing values of $m$. If we consider the ratio between $P_{M,(m+1) \cdot 1}(m+1)$ and $P_{M, m \cdot 1}(m)$, we observe that, for $m \geq 8$, it oscillates between the values 0.6 and 0.7. Thus we can give an upper and a lower estimate for the least value of $m$ giving 5 -reformed decks. Supposing

$$
0.6 \cdot P_{M, m \cdot 1}(m) \leq P_{M,(m+1) \cdot 1}(m+1) \leq 0.7 \cdot P_{M, m \cdot 1}(m) \quad, \quad \forall m \geq 15,
$$

we can expect the first 5 -reformed decks for $18 \leq m \leq 24$.

On the other hand, observing that the ratio between $N_{M,(m+1) \cdot 1}$ and $N_{M, m \cdot 1}$ grows with $m$ and that

$$
\frac{N_{M, 15 \cdot 1}}{N_{M, 14 \cdot 1}} \sim 9.5
$$

we have chances to find 5-reformed decks if the ratio $\frac{N_{M,(m+1) \cdot 1}}{N_{M, m \cdot 1}}$ exceeds the threshold value 11.5, for $m \geq 15$. In this case, we could expect the first 5-reformed permutations for $18 \leq m \leq 21$, an estimate which is in good agreement with the previous one.

The improvement of the results up to $m=16$, or even $m=17$, by means, for example, of parallel calculus, could give more refined estimates for $P_{M, m \cdot 1}(m)$ and of $N_{M, m \cdot 1}$ and, consequently, for the first value of $n$ giving a 5 -reformed deck.

Moreover, the extension of our analysis to $s>1$ can confirm the argument we proposed above, concerning the relationship between $P_{M, m \cdot s}(m \cdot s)$ and the appearance of $k$-reformed decks.

In fact, passing to "multiseed" Mousetrap, we have found, at most, 3-reformed decks. Also in this case, in order to look for 4-reformed permutations, we can compare the growth rate of $N_{M, m \cdot s}$ and the decrease rate of $P_{M, m \cdot s}(m \cdot s)$. In the most advanced cases we have examined (i.e., $m=8, s=2 ; m=6, s=$ $3 ; m=5, s=2)$, we found a large number of 3-reformed decks. Though $P_{M, m \cdot s}(m \cdot s)$ is rapidly decreasing, we can expect 4-reformed decks already for $s=2,9 \leq m \leq 11$ and $s=3,7 \leq m \leq 9$; for $s=4$, we are not able to predict when we will find 4-reformed decks, because we have not yet a sufficient amount of data to understand the behavior of $P_{M, 4 \cdot m}(4 \cdot m)$.

Let us observe that, for $m=3, s=4$, we found the first (and, up to now, unique) non trivial 1-cycle: 111122322333 . Consequently, the second part of question 5) receives a positive answer, but only in a "multiseed" framework, while a negative answer is highly probable for $s=1$.

Passing to Modular Mousetrap, we will need some terminology, in order to distinguish the different situations we will deal with.

I will distinguish the reformed decks into two different categories:

i) $k$-reformed decks;

ii) $k$-cycles, i.e., decks which are reformed $k$ times and such that the $k$-th deck coincides with one of the previous reformations.

If the $k$-th reformation coincides the $h$-th reformation $(1 \leq h<k)$, we will divide the total $k$-cycle into two parts: 
i) a $h$-pre-loop, where there is a sequence of $h$ reformations;

ii) a $(k-h)$-loop, starting from the $h$-th reformation and stopping at the $k$-th reformation, which coincides with the $h$-th one.

Guy and Nowakowski analyzed all the reformed permutations for $s=1, m \leq 5$. Clearly, this analysis cannot be easily performed for greater values of $m$. In fact, for $m=6$, they consider only decks where the first card is an "ace".

We have improved their results to many more cases and to $1<s \leq 4$, as shown in Tables $5-8$.

Thanks to the high winning probability, in particular if $n$ is prime, the game Modular Mousetrap has produced many interesting and intriguing results. The reason why we can obtain very long sequences and cycles in Modular Mousetrap must be found in the fact that, as already remarked, in this game, when $n$ is prime, we have always a winning deck or a derangement and that the probability to yield a winning deck is very high (see Table 8). Consequently, in this case it is very easy to obtain, from a deck, a reformed one. Due to the highly increasing number of permutations when $m$ grows, we were able to study all the decks in Modular Mousetrap, for $s=1$, only up to $m=12$.

But since we expect to yield many more interesting results whenever $n$ is prime, we have also examined the first 100 millions reformed decks for $s=1$ and $m=13,17,19$.

The most complete and rich investigation has been performed for $s=1, m=11$, for which we analyzed all the decks, by means of the "eulerian" technique. Table 5 shows the huge increase of the number of cycles, with respect to smaller values of $m$.

In this case, as shown in Table 9, we found six 203-cycles, obtained starting from the decks

$$
\begin{aligned}
& 1115826947103 ; \quad 1115826910743 ; \quad 1115926108743 ; \\
& 1115826497103 ; \quad 1115826491037 ; \quad 1115926810743 .
\end{aligned}
$$

For example, the deck

$$
1115826947103
$$

after 137 reformations, enters in a loop, because the 203-th reformed deck is identical to the 137 -th deck.

Furthermore, the deck

$$
1117421039568
$$

yields a 156-pre-loop, which is, up to now, the longest one. Then, it enters in a 1-loop, giving a total 157-cycle.

Curiously, for $m=11$ the decks gave only $1,2,3,4,14,15$ and 66-loops. The number of decks entering in a 66 -loop is very high: 1,701,937.

We can understand the richness of Modular Mousetrap considering that in our investigations we obtained the longest $k$-reformed deck for $s=1, m=19$, though we analyzed only 100 millions decks among all the 19 ! 1.22 $\cdot 10^{17}$ permutations, while, for $s=1, m=11$, we arrived "only" at a 41-reformed deck analyzing all the $25,232,230$ decks.

Consequently, it is highly probable that the record attained by the deck

$$
11541914181631971013171215862 \text {, }
$$

which is 45-times reformable, could be improved, if we would study more cases for $s=1, m$ prime and $m \geq 13$. The case $m=13$ is still under investigation. 
Concerning the 1-cycles, for $s=1$ there is no evidence of other cycles than the trivial one $\left(\begin{array}{llll}1 & 2 & \cdots & m\end{array}\right)$. When we pass to "multiseed" Modular Mousetrap, we have not only the trivial 1-cycle

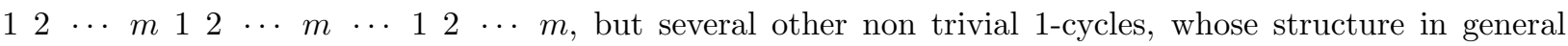
seems not to have any regularity: for example, the decks

$1122 ; 131223 ; 14312234 ; 163451223456 ; 1345122345$;

2765713243456 ; 26657113243457 ; 25657113243467 ; 27651135243467 ; 36324451712567 ; 13456712234567 ; 13532146524677 ;

$111222 ; 131223123 ; 111223323$ etc.

give 1-cycles.

Up to now, we have achieved the greatest number of 1-cycles for $s=3, m=5$ and for $s=4, m=4$, where we found ten different 1-cycles. Since the two cases were the most advanced in our studies, we can suppose that we could obtain greater numbers of non trivial 1-cycles, if we could continue our analysis for higher values of $m$.

While for Mousetrap the existence of sequences of arbitrary length is still an open problem, the explosion of the number of $k$-cycles and $k$-reformed decks, already for $s=1$, allows us to give a partial answer to questions 5) and 6) for Modular Mousetrap, which is shown in Table 13. The results reported in this table seem to suggest a positive answer to the first part of question 6).

Due to the difficulty to report all the results, we have built the web page

http://www.dmmm.uniroma1.it/ bersani/mousetrap.html

where we report all the results for Modular Mousetrap and show the numbers of loops, pre-loops, total cycles and reformed decks, for the different values of $m$ and $s$ we investigated. The page is still under construction and many documents are still written in Italian language, but the meaning of the results is clear.

We extended the study of reformed decks to the game $(H L M)^{2} N$, too.

We can repeat the considerations related to the connection between the appearance of $k$-reformed decks and the probability to obtain the best record, which we indicate with $P_{\max }:=P\left(C_{\max }\right)$. Knowing the low probability to have winning decks at $(H L M)^{2} N$ (see [2, Table 5]), we cannot expect to easily yield $k$-reformed decks, with $k \geq 2$, at this game.

In fact, Table 14 shows that, excluding the trivial 1-cycles $11 \cdots 1$ and $11 \cdots 122 \cdots 2$, there is no evidence of $k$-reformed decks $(k \geq 2)$, apart from the unique, very special case $m=2, s=4$, where we attained the following four 2-times reformable decks:

$$
22221111 ; 12222111 ; 11222211 ; 11122221 \text {. }
$$

The very fast decrease of $P_{\text {max }}$, when $m$ grows, seems not to allow us to obtain 2-reformed decks in other cases.

Thus, we have focussed on the reformed decks satisfying (WC), instead of (SC). As shown in Table 15, we have found new 2-reformed decks only in the cases $s=4,4 \leq m$, where the growth rate of the number of total reformed decks is sufficiently high to compensate the decrease of the probability $P_{\max }(W C)$ to yield decks satisfying (WC). Since $P_{\max , 6 \cdot 4}(W C) \sim 4 \cdot 10^{-8}$, it is an open question if it is possible to yield 3-reformed decks for higher values of $m$.

The case $s=1$ has been studied only for $1 \leq m \leq 4$, because, as shown by the author [2, Table 6], (WC) is satisfied only for these values of $m$. 
For $m=1$, the unique deck 1 is a 1-cycle. For $m=2$, we have only the 1 -cycle 12 . For $m=3$ we have two 1-reformable decks: 132 and 321 . For $m=4$ we have only the 1-reformable deck 2134 .

\section{Conclusions and further developments}

The "eulerian" technique introduced by the author [2] has proved to be very powerful for the study of the games He Loves Me, He loves Me Not, Mousetrap and Modular Mousetrap, in particular, for what concerns the reformed permutations. Clearly, it can give only the number of winning decks, without any possibility to arrive at a closed formula. But the complexity of the game studied is so high that it is very hard to expect to find general closed formulas. In fact, as already remarked, only partial results have been obtained in the previous literature.

The contraindication for this "eulerian" method is related to disk usage problems: in order to build all the strings of length $(k+1)$, the program needs to store all the strings of length $k$.

Even if we should not be interested on the storage of all the winning decks, but only on their number, it is however necessary to store all the winning $(n-1)$-strings. In the game Mousetrap, in the case $m=15, s=1$, the storage of all the winning 15-decks needed a 42.3 GB memory, while the storage of all the winning 14strings needed a 70.6 GB memory.

Certainly, the usage of more powerful computers and of parallel calculus, to which the technique is easily adaptable, or, at least (as actually done playing $(H L M)^{2} N$ in the cases $m=9, s=3$ and $m=7, s=4$ and Mousetrap in the case $m=15, s=1$ ), the storage of all the strings of the same length $k$ in several data subfiles, which can be separately processed, could help the search for all the reformed decks for higher values of $m$ and/or $s$. Less disk usage expensive computer methods or other improvements could help, too.

Up to now, the "eulerian" technique seems to be the unique capable to give more complete answers to questions 1) - 6). No one of them has received yet a definitive answer. In particular, finding 5-reformed decks at Mousetrap could bring to conjecture that, for increasing values of $m$, it is possible to yield $k$ reformed decks for every value of $k$ (question 4)). As already observed, the answer strongly depends on the competition between the growth rate of the number of total reformed decks and the decrease rate of $P_{\max }$, when $m$ grows. It could be very useful to study the game for increasing values of $m$, by means of the "eulerian" technique, implemented in a parallel calculus framework. The improvement of the technique, mainly concerning the memory saving problems, could lead to more satisfactory results.

For example, it is highly probable that the record attained at Modular Mousetrap by the deck

$$
11541914181631971013171215862
$$

which is 45-times reformable, could be improved, if we would study more cases for $s=1, m$ prime and $m \geq 13$.

In order to encourage further suggestions to improve the memory saving and the algorithm we implemented, we insert all the FORTRAN files used for our researches in our web page

http://www.dmmm.uniroma1.it/ bersani/mousetrap.html together with all the results for Modular Mousetrap. Since the page is still under construction, the comments in the FORTRAN files are still written in Italian. However, waiting for their translation in English, I am at disposal of everyone who would like to collaborate to this research, in order to explain the passages in the FORTRAN files. 
Acknowledgments I am deeply grateful to Proff. N. J. A. Sloane and K. S. Chua, for their information on the most recent results on reformed decks and to all the people who have given me even simple suggestions to improve the computer programs through which I have implemented the "eulerian" technique.

I will highly appreciate every information on improvements of the results reported in this paper and/or of the computer programs to obtain them. 


\section{REFERENCES}

[1] A. M. Bersani, Aspetti combinatori di una classe di problemi di rencontre: risultati classici e sviluppi recenti, preprint Me.Mo.Mat.

[2] A. M. Bersani, An "eulerian" approach to a class of matching problems, preprint Me.Mo.Mat.

[3] A. Cayley, A problem in permutations, Quart. J. Pure Appl. Math. 1 (1857), 79.

[4] A. Cayley, On the game of Mousetrap, Quart. J. Pure Appl. Math. 15 (1878), 8 - 10.

[5] K. S. Chua, private communication.

[6] M. Fréchet, Les probabilités associées a un système d'événements compatibles et dépendants - Seconde partie: cas particuliers et applications, Hermann and C., Paris, 1943.

[7] R. K. Guy, Mousetrap, $\S E 37$ in Unsolved Problems in Number Theory, second edition, Springer-Verlag, New York, 1994, pp. 237 - 238.

[8] R. K. Guy and R. Nowakowski, Mousetrap, in D. Miklós, V.T. Sós and T. Szonyi, eds., Combinatorics, Paul Erdős is Eighty, vol. 1, János Bolyai Mathematical Society, Budapest, 1993, pp. 193 - 206.

[9] R. K. Guy and R. Nowakowski, Unsolved Problems - Mousetrap, Amer. Math. Monthly 101 (1994), $1007-1008$.

[10] R. K. Guy and R. Nowakowski, Monthly Unsolved Problems, 1969 - 1995, Amer. Math. Monthly 102 (1995), $921-926$.

[11] D. J. Mundfrom, A problem in permutations: the game of Mousetrap, European J. Combin. 15 (1994), $555-560$.

[12] A. Pompili, Il metodo MONTE CARLO per l'analisi di un solitario, http://xoomer.virgilio.it/vdepetr/Art/Text22.htm.

[13] N. J. A. Sloane, A Handbook of integer sequences, Academic Press, San Diego, 1973.

[14] N. J. A. Sloane, S. Plouffe, The encyclopedia of integer sequences, Academic Press, San Diego, 1995.

[15] N. J. A. Sloane, The On-Line Encyclopaedia of Integer Sequences, http://www.research.att.com/ njas/sequences/ .

[16] N. J. A. Sloane, private communication.

[17] A. Steen, Some formulae respecting the Game of Mousetrap, Quart. J. Pure Appl. Math. 15 (1878), $230-241$.

OEIS: A000166, A001563, A002467, A002468, A002469, A007709, A007710, A007711, A007712, A018931, A018932, A018933, A028305, A028306, A055459, A067950 


\begin{tabular}{|c||c|c|c|c|c|c|c|}
\hline & unreformed & 1-reformed & 2-reformed & 3-reformed & 4-reformed & 1-cycles & total reformed \\
\hline \hline$m=1$ & 0 & 0 & 0 & 0 & 0 & 1 & 1 \\
\hline$m=2$ & 1 & 0 & 0 & 0 & 0 & 1 & 1 \\
\hline$m=3$ & 4 & 2 & 0 & 0 & 0 & 0 & 0 \\
\hline$m=4$ & 18 & 4 & 2 & 0 & 0 & 0 \\
\hline$m=5$ & 105 & 14 & 1 & 0 & 0 & 0 & 15 \\
\hline$m=6$ & 636 & 72 & 11 & 1 & 0 & 0 & 0 \\
\hline$m=7$ & 4710 & 316 & 14 & 0 & 0 & 0 & 330 \\
\hline$m=8$ & 38508 & 1730 & 81 & 1 & 0 & 0 & 0 \\
\hline$m=9$ & 352,902 & 9728 & 242 & 8 & 0 & 9978 \\
\hline$m=10$ & $3,563,297$ & 64330 & 1142 & 31 & 0 & 0 & 65503 \\
\hline$m=11$ & $39,467,081$ & 444,890 & 4771 & 56 & 2 & 0 & 449,719 \\
\hline$m=12$ & $475,326,930$ & $3,645,441$ & 29009 & 219 & 1 & 0 & $3,674,670$ \\
\hline$m=13$ & $6,198,134,207$ & $28,758,111$ & 127,876 & 605 & 1 & 0 & $28,886,593$ \\
\hline$m=14$ & $86,912,048,471$ & $265,434,293$ & 805,947 & 2485 & 4 & 0 & $266,242,729$ \\
\hline$m=15$ & $1,305,146,666,727$ & $2,522,822,881$ & $4,868,681$ & 9697 & 14 & 0 & $2,527,701,273$ \\
\hline
\end{tabular}

Table 1

Number of unreformed and reformed decks at Mousetrap for $s=1$. The values for $1 \leq m \leq 9$ were reported by Guy and Nowakowski [8]. The values for $10 \leq m \leq 13$ were reported by Chua [5] and Sloane [15]. There is no evidence of 5-reformed decks, in any case we have examined. The first column extends the sequence [15] A007711; the second column extends [15] A007712; the third column extends [15] A055459; the fourth column extends [15] A067950; the last column extends [15] A007709.

\begin{tabular}{|c||c|c|c|c|c|c|}
\hline & unreformed & 1-reformed & 2-reformed & 3-reformed & 1-cycles & total reformed \\
\hline \hline$m=1$ & 1 & 0 & 0 & 0 & 1 & 1 \\
\hline$m=2$ & 3 & 2 & 0 & 0 & 1 & 3 \\
\hline$m=3$ & 78 & 12 & 0 & 0 & 0 & 12 \\
\hline$m=4$ & 2373 & 132 & 14 & 1 & 0 & 147 \\
\hline$m=5$ & 111,078 & 2270 & 51 & 1 & 0 & 2322 \\
\hline$m=6$ & $7,412,771$ & 70766 & 857 & 6 & 0 & 71629 \\
\hline$m=7$ & $678,866,142$ & $2,207,169$ & 7071 & 18 & 0 & $2,214,258$ \\
\hline$m=8$ & $81,611,419,132$ & $118,065,748$ & 162,871 & 249 & 0 & $118,228,868$ \\
\hline
\end{tabular}

Table 2

Number of unreformed and reformed decks at Mousetrap for $s=2$. There is no evidence of 4-reformed decks, in any case we have examined. 


\begin{tabular}{|c||c|c|c|c|c|c|}
\hline & unreformed & 1-reformed & 2-reformed & 3-reformed & 1-cycles & total reformed \\
\hline \hline$m=1$ & 0 & 0 & 0 & 0 & 1 & 1 \\
\hline$m=2$ & 16 & 3 & 0 & 0 & 1 & 4 \\
\hline$m=3$ & 1590 & 86 & 4 & 0 & 0 & 90 \\
\hline$m=4$ & 364,368 & 5148 & 84 & 2 & 0 & 5232 \\
\hline$m=5$ & $167,691,958$ & 474,658 & 1384 & 1 & 0 & 476,042 \\
\hline$m=6$ & $137,113,427,648$ & $111,570,619$ & 89649 & 84 & 0 & $111,660,352$ \\
\hline
\end{tabular}

Table 3

Number of unreformed and reformed decks at Mousetrap for $s=3$. There is no evidence of 4-reformed decks, in any case we have examined.

\begin{tabular}{|c||c|c|c|c|c|c|}
\hline & unreformed & 1-reformed & 2-reformed & 3-reformed & 1-cycles & total reformed \\
\hline \hline$m=1$ & 0 & 0 & 0 & 0 & 1 & 1 \\
\hline$m=2$ & 55 & 11 & 4 & 0 & 1 & 15 \\
\hline$m=3$ & 33975 & 639 & 35 & 0 & 1 & 675 \\
\hline$m=4$ & $62,852,931$ & 209,411 & 658 & 0 & 0 & 210,069 \\
\hline$m=5$ & $305,420,859,119$ & $119,321,646$ & 54210 & 25 & 0 & $119,375,881$ \\
\hline
\end{tabular}

Table 4

Number of unreformed and reformed decks at Mousetrap for $s=4$. In the case $m=3$ we find for the first time a non-trivial 1-cycle: 111122322333. There is no evidence of 4-reformed decks, in any case we have examined. 


\begin{tabular}{|c||c|c|c|c|}
\hline & unreformed & k-reformed & cycles & total reformed \\
\hline \hline$m=1$ & 0 & 0 & 1 & 1 \\
\hline$m=2$ & 1 & 0 & 2 & 1 \\
\hline$m=3$ & 2 & 2 & 5 & 4 \\
\hline$m=4$ & 15 & 4 & 39 & 9 \\
\hline$m=5$ & 44 & 37 & 20 & 76 \\
\hline$m=6$ & 530 & 170 & 850 & 3186 \\
\hline$m=7$ & 1854 & 2336 & 274 & 11351 \\
\hline$m=8$ & 28969 & 11077 & 2815 & 132,684 \\
\hline$m=9$ & 230,196 & 129,869 & 671 & 884,371 \\
\hline$m=10$ & $2,744,429$ & 883,700 & $3,702,258$ & $25,232,230$ \\
\hline$m=11$ & $14,684,570$ & $21,529,972$ & 1352 & $50,436,488$ \\
\hline$m=12$ & $428,565,112$ & $50,435,136$ & under investigation & $3,936,227,868$ \\
\hline$m=13$ & $2,290,792,932$ & under investigation & & \\
\hline
\end{tabular}

Table 5

Number of unreformed and reformed decks at Modular Mousetrap for $s=1$. The values for $1 \leq m \leq 5$ were reported by Guy and Nowakowski [8]. The case $m=13$ is still under investigation. Since in this game, for $s=1$ and $m$ prime, a deck can only either win or give a derangement, we can obtain the number of unreformed decks by a theoretical point of view, because coincides with the number of derangements (see sequences [15] A000166 and A002467 and formula (2.10)).

\begin{tabular}{|c||c|c|c|c|}
\hline & unreformed & k-reformed & cycles & total reformed \\
\hline \hline$m=1$ & 0 & 0 & 1 & 1 \\
\hline$m=2$ & 1 & 0 & 5 & 5 \\
\hline$m=3$ & 30 & 39 & 21 & 60 \\
\hline$m=4$ & 1338 & 1027 & 155 & 1182 \\
\hline$m=5$ & 50337 & 57581 & 5482 & 63063 \\
\hline$m=6$ & $5,687,050$ & $1,796,111$ & 1239 & $1,797,350$ \\
\hline$m=7$ & $316,508,244$ & $364,074,715$ & 497,441 & $364,572,156$ \\
\hline
\end{tabular}

Table 6

Number of unreformed and reformed decks at Modular Mousetrap for $s=2$. 


\begin{tabular}{|c||c|c|c|c|}
\hline & unreformed & k-reformed & cycles & total reformed \\
\hline \hline$m=1$ & 0 & 0 & 1 & 1 \\
\hline$m=2$ & 1 & 0 & 19 & 19 \\
\hline$m=3$ & 599 & 615 & 466 & 1081 \\
\hline$m=4$ & 196,547 & 161,772 & 11281 & 173,053 \\
\hline$m=5$ & $81,531,697$ & $86,339,122$ & 297,181 & $86,636,303$ \\
\hline
\end{tabular}

Table 7

Number of unreformed and reformed decks at Modular Mousetrap for $s=3$.

\begin{tabular}{|c||c|c|c|c|}
\hline & unreformed & k-reformed & cycles & total reformed \\
\hline \hline$m=1$ & 0 & 0 & 1 & 1 \\
\hline$m=2$ & 1 & 0 & 69 & 69 \\
\hline$m=3$ & 11752 & 15466 & 7432 & 22898 \\
\hline$m=4$ & $33,420,815$ & $29,381,680$ & 260,505 & $29,642,185$ \\
\hline
\end{tabular}

Table 8

Number of unreformed and reformed decks at Modular Mousetrap for $s=4$. 


\begin{tabular}{|c||c|c|c|c|c|}
\hline & MAX $k$-reformed & MAX $k$-cycle & MAX $k$-pre-loop & MAX $k$-loop & number of 1-cycles \\
\hline \hline$m=1$ & 0 & 1 & 0 & 1 & 1 \\
\hline$m=2$ & 0 & 1 & 0 & 1 & 1 \\
\hline$m=3$ & 2 & 2 & 1 & 1 & 1 \\
\hline$m=4$ & 2 & 3 & 2 & 1 & 1 \\
\hline$m=5$ & 3 & 5 & 4 & 2 & 1 \\
\hline$m=6$ & 5 & 5 & 4 & 1 & 1 \\
\hline$m=7$ & 10 & 19 & 18 & 2 & 1 \\
\hline$m=8$ & 8 & 9 & 8 & 2 & 1 \\
\hline$m=9$ & 13 & 13 & 11 & 2 & 1 \\
\hline$m=10$ & 10 & 6 & 5 & 3 & 1 \\
\hline$m=11$ & 41 & 203 & 156 & 66 & 1 \\
\hline$m=12$ & 8 & 7 & 6 & 1 & $\geq 1$ \\
\hline$m=13$ & $\geq 43$ & $\geq 22$ & $\geq 20$ & $\geq 3$ & $\geq 1$ \\
\hline$m=17$ & $\geq 39$ & $\geq 19$ & $\geq 17$ & $\geq 2$ & $\geq 1$ \\
\hline$m=19$ & $\geq 45$ & $\geq 1$ & $\geq 0$ & $\geq 1$ & \\
\hline
\end{tabular}

Table 9

Maximal values for the different cases of reformed decks ( $k$-reformed, loops, pre-loops, $k$-cycles) for Modular Mousetrap for $s=1$. In the last column we report the number of 1 -cycles. For every value of $m$, the permutation $\{1,2,3, \cdots, m-1, m\}$ gives a 1-cycle. There is no evidence for other (non trivial) 1-cycles. In the cases $m=13,17,19$ we have examined only the first 100 millions winning decks, because the total number of decks to be examined it too high. Since 13,17,19 are prime numbers, it is highly probable that the maximal values of different reformed decks can improve the values obtained for $m=11$.

\begin{tabular}{|c||c|c|c|c|c|}
\hline & MAX $k$-reformed & MAX $k$-cycle & MAX $k$-pre-loop & MAX $k$-loop & number of 1-cycles \\
\hline \hline$m=1$ & 0 & 1 & 0 & 1 & 1 \\
\hline$m=2$ & 0 & 3 & 2 & 1 & 2 \\
\hline$m=3$ & 4 & 3 & 2 & 1 & 2 \\
\hline$m=4$ & 9 & 7 & 5 & 2 & 2 \\
\hline$m=5$ & 14 & 15 & 14 & 3 & 2 \\
\hline$m=6$ & 13 & 7 & 6 & 2 & 2 \\
\hline$m=7$ & 29 & 24 & 23 & 2 & 8 \\
\hline
\end{tabular}

Table 10

Maximal values for the different cases of reformed decks ( $k$-reformed, loops, pre-loops, $k$-cycles) for Modular Mousetrap for $s=2$. In the last column we report the number of 1 -cycles. For every value of $m$, the permutation $\{1,2,3, \cdots, m-1, m, 1,2,3, \cdots, m-1, m\}$ gives a 1-cycle. However, in this case we yield other (non trivial) 1-cycles. 


\begin{tabular}{|c||c|c|c|c|c|}
\hline & MAX $k$-reformed & MAX $k$-cycle & MAX $k$-pre-loop & MAX $k$-loop & number of 1-cycles \\
\hline \hline$m=1$ & 0 & 1 & 0 & 1 & 1 \\
\hline$m=2$ & 0 & 4 & 2 & 2 & 2 \\
\hline$m=3$ & 8 & 10 & 7 & 6 & 3 \\
\hline$m=4$ & 17 & 12 & 10 & 2 & 5 \\
\hline$m=5$ & 30 & 19 & 18 & 4 & 10 \\
\hline
\end{tabular}

Table 11

Maximal values for the different cases of reformed decks ( $k$-reformed, loops, pre-loops, $k$-cycles) for Modular Mousetrap for $s=3$. In the last column we report the number of 1 -cycles. For every value of $m$, the permutation $\{1,2,3, \cdots, m-1, m, \cdots 1,2,3, \cdots, m-1, m\}$ gives a 1-cycle. However, in this case we yield other (non trivial) 1-cycles.

\begin{tabular}{|c||c|c|c|c|c|}
\hline & MAX $k$-reformed & MAX $k$-cycle & MAX $k$-pre-loop & MAX $k$-loop & number of 1-cycles \\
\hline \hline$m=1$ & 0 & 1 & 0 & 1 & 1 \\
\hline$m=2$ & 0 & 5 & 3 & 3 & 3 \\
\hline$m=3$ & 15 & 12 & 11 & 4 & 6 \\
\hline$m=4$ & 28 & 17 & 14 & 3 & 10 \\
\hline
\end{tabular}

Table 12

Maximal values for the different cases of reformed decks ( $k$-reformed, loops, pre-loops, $k$-cycles) for Modular Mousetrap for $s=4$. In the last column we report the number of 1 -cycles. For every value of $m$, the permutation $\{1,2,3, \cdots, m-1, m, \cdots 1,2,3, \cdots, m-1, m\}$ gives a 1-cycle. However, in this case we yield other (non trivial) 1-cycles.

\begin{tabular}{|c||c|c|c|}
\hline & $\begin{array}{c}\text { least value of } n \\
\text { yielding a } k \text {-loop }\end{array}$ & $\begin{array}{c}\text { least value of } n \\
\text { yielding a } k \text {-cycle }\end{array}$ & $\begin{array}{c}\text { least value of } n \\
\text { yielding a } k \text {-reformed deck }\end{array}$ \\
\hline \hline$k=1$ & 1 & 1 & 3 \\
\hline$k=2$ & 5 & 3 & 3 \\
\hline$k=3$ & 10 & 4 & 5 \\
\hline$k=4$ & 11 & 5 & 6 \\
\hline$k=5$ & - & 5 & 6 \\
\hline
\end{tabular}

Table 13

Least value of $n$ which yields a $k$-loop, or a $k$-cycle, or a $k$-reformed deck at Modular Mousetrap, with $s=1$. The table is based on the results obtained for $m \leq 11$. Let us observe that for $m=11$ we found even longer $k$-loops, corresponding only to the values $k=14,15,66$. The first value of $n$ yielding $k$-cycles, for $6 \leq k \leq 19$, is 7; the first value of $n$ yielding $k$-cycles, for $20 \leq k \leq 203$, is 11 . The first value of $n$ yielding $k$-reformed decks, for $6 \leq k \leq 10$, is 7 ; the first value of $n$ yielding $k$-reformed decks, for $11 \leq k \leq 13$, is 9 ; the first value of $n$ yielding $k$-reformed decks, for $14 \leq k \leq 41$, is 11 . 


\begin{tabular}{|c|c|c|c|}
\hline & $s=2(\mathrm{SC})$ & $s=3(\mathrm{SC})$ & $s=4(\mathrm{SC})$ \\
\hline$m=1$ & $\begin{array}{ll}1 & 1-\text { cycle } \\
1 & \text { total reformed }\end{array}$ & $\begin{array}{ll}1 & 1-\text { cycle } \\
1 & \text { total reformed }\end{array}$ & $\begin{array}{ll}1 & 1-\text { cycle } \\
1 & \text { total reformed }\end{array}$ \\
\hline$m=2$ & $\begin{array}{ll}1 & 1-\text { cycle } \\
2 & 1-\text { reformed } \\
3 & \text { total reformed }\end{array}$ & $\begin{array}{ll}1 & 1-\text { cycle } \\
3 & 1-\text { reformed } \\
4 & \text { total reformed }\end{array}$ & $\begin{array}{l}1 \quad 1-\text { cycle } \\
10 \quad 1 \text {-reformed } \\
4 \quad 2-\text { reformed } \\
15 \quad \text { total reformed }\end{array}$ \\
\hline$m=3$ & only unreformed & $\begin{array}{ll}4 & 1-\text { reformed } \\
4 & \text { total reformed }\end{array}$ & $\begin{array}{ll}5 & 1-\text { reformed } \\
5 & \text { total reformed }\end{array}$ \\
\hline$m=4$ & only unreformed & $\begin{array}{cl}9 & 1-\text { reformed } \\
9 & \text { total reformed }\end{array}$ & $\begin{array}{ll}229 & 1-\text { reformed } \\
229 & \text { total reformed }\end{array}$ \\
\hline$m=5$ & only unreformed & $\begin{array}{l}63 \text { - } 1 \text {-reformed } \\
63 \text { total reformed }\end{array}$ & $\begin{array}{cl}10568 & 1-\text { reformed } \\
10568 & \text { total reformed }\end{array}$ \\
\hline$m=6$ & $\begin{array}{ll}1 & 1-\text { reformed } \\
1 & \text { total reformed }\end{array}$ & $\begin{array}{ll}1177 & 1-\text { reformed } \\
1177 & \text { total reformed }\end{array}$ & $\begin{array}{ll}1,212,483 & 1-\text { reformed } \\
1,212,483 & \text { total reformed }\end{array}$ \\
\hline$m=7$ & $\begin{array}{ll}7 & 1-\text { reformed } \\
7 & \text { total reformed }\end{array}$ & $\begin{array}{ll}36144 & 1-\text { reformed } \\
36144 & \text { total reformed }\end{array}$ & $\begin{array}{ll}411,488,689 & 1-\text { reformed } \\
411,488,689 & \text { total reformed }\end{array}$ \\
\hline$m=8$ & $\begin{array}{ll}8 & 1-\text { reformed } \\
8 & \text { total reformed }\end{array}$ & $\begin{array}{ll}1,677,968 & 1-\text { reformed } \\
1,677,968 & \text { total reformed }\end{array}$ & \\
\hline$m=9$ & $\begin{array}{ll}105 & 1-\text { reformed } \\
105 & \text { total reformed }\end{array}$ & $\begin{array}{ll}127,255,522 & 1-\text { reformed } \\
127,255,522 & \text { total reformed }\end{array}$ & \\
\hline$m=10$ & $\begin{array}{l}656 \quad 1-\text { reformed } \\
656 \text { total reformed }\end{array}$ & & \\
\hline$m=11$ & $\begin{array}{ll}6745 & 1-\text { reformed } \\
6745 & \text { total reformed }\end{array}$ & & \\
\hline$m=12$ & $\begin{array}{ll}76823 & 1-\text { reformed } \\
76823 & \text { total reformed }\end{array}$ & & \\
\hline$m=13$ & $\begin{array}{ll}986,994 & 1-\text { reformed } \\
986,994 & \text { total reformed }\end{array}$ & & \\
\hline$m=14$ & $\begin{array}{ll}17,175,636 & 1-\text { reformed } \\
17,175,636 & \text { total reformed }\end{array}$ & & \\
\hline
\end{tabular}

Table 14

Number of reformed decks satisfying $(\mathrm{SC})$ at $(H L M)^{2} N$. Since the value of $P_{\max }$ decreases very quickly when $m$ grows, we cannot expect 2-reformed decks, apart from the case $m=2, s=4$. 


\begin{tabular}{|c|c|c|c|}
\hline & $s=2(\mathrm{WC})$ & $s=3(\mathrm{WC})$ & $s=4(\mathrm{WC})$ \\
\hline \multirow{2}{*}{$m=1$} & $111-$ cycle & $111-$ cycle & $111-$ cycle \\
\hline & 1 total reformed & 1 total reformed & 1 total reformed \\
\hline \multirow{4}{*}{$m=2$} & & & $11-$ cycles \\
\hline & 21 -reformed & 41 -reformed & 101 -reformed \\
\hline & 3 total reformed & 5 total reformed & 42 -reformed \\
\hline & o corat rejormed & o corar tejortmeda & 15 total reformed \\
\hline \multirow{2}{*}{$m=3$} & 61 -reformed & 301 -reformed & 1601 -reformed \\
\hline & 6 total reformed & 30 total reformed & 160 total reformed \\
\hline \multirow[b]{2}{*}{$m=4$} & 101 -reformed & 2781 -reformed & $74101-$ reformed \\
\hline & 10 total reformed & 278 total reformed & 12 -reformed \\
\hline \multirow{3}{*}{$m=5$} & & & 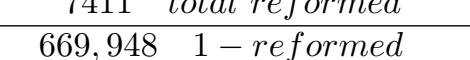 \\
\hline & $56 \quad 1$-reformed & 5027 1-reformed & 42 -reformed \\
\hline & 56 total reformed & 5027 total reformed & 669,952 total reformed \\
\hline \multirow{2}{*}{$m=6$} & 2001 -reformed & $132,437 \quad 1$-reformed & $\begin{array}{l}133,085,352 \quad 1 \text {-reformed } \\
152-\text { - }\end{array}$ \\
\hline & 200 total reformed & 132,437 total reformed & $\begin{array}{l}133,085,367 \text { total reformed } \\
132-\text { rejormea }\end{array}$ \\
\hline \multirow{2}{*}{$m=7$} & 10941 -reformed & $6,131,753 \quad 1-$ reformed & \\
\hline & 1094 total reformed & $6,131,753$ total reformed & \\
\hline \multirow{2}{*}{$m=8$} & $7016 \quad 1$-reformed & & \\
\hline & 7016 total reformed & & \\
\hline \multirow{2}{*}{$m=9$} & $55661 \quad 1-$ reformed & & \\
\hline & 55661 total reformed & & \\
\hline \multirow{2}{*}{$m=10$} & $586,810 \quad 1-$ reformed & & \\
\hline & 586,810 total reformed & & \\
\hline \multirow{2}{*}{$m=11$} & $7,340,841 \quad 1-$ reformed & & \\
\hline & $7,340,841$ total reformed & & \\
\hline \multirow{2}{*}{$m=12$} & $114,616,993 \quad 1-$ reformed & & \\
\hline & $114,616,993$ total reformed & & \\
\hline
\end{tabular}

Table 15

Number of reformed decks satisfying (WC) at $(H L M)^{2} N$. For $s=4$, since the number of reformed decks grows very quickly, it is possible to yield 2-reformed decks. 